\title{
Delta-secretase triggers Alzheimer's disease pathologies in wild-type hAPP/hMAPT double transgenic mice
}

\author{
Zhourui Wu $\mathbb{B}^{1,2,3}$, Xia Liu', Liming Cheng ${ }^{2,3}$ and Keqiang Ye $\mathbb{B}^{1}$
}

\begin{abstract}
Alzheimer's disease (AD) is the most common neurodegenerative disease with multifactorial pathologies including $A \beta$ containing senile plaques and neurofibrillary tangles (NFT) consisted of aggregated Tau. Most of the AD patients are sporadic and the familial mutation hereditary patients are composed only $1 \%$ of all cases. However, the current AD mouse models employ mutated APP, PS1, or even Tau mutant, in order to display a portion of AD pathologies. Deltasecretase (legumain, or asparaginyl endopeptidase, AEP) simultaneously cleaves both APP and Tau and augments A $\beta$ production and Tau hyperphosphorylation and aggregation, contributing to AD pathogenesis. Here we show that $\delta$-secretase is sufficient to promote prominent AD pathologies in wild-type hAPP/hMAPT double transgenic mice. We crossed hAPP I5 mice and hMAPT mice to generate double transgenic mouse model carrying both human wild-type APP and Tau. Compared to the single transgenic parents, these double transgenic mice demonstrated AD-related pathologies in one-year-old hAPP/hMAPT mice. Notably, overexpression of $\delta$-secretase in hAPP/hMAPT doubletransgenic mice evidently accelerated enormous senile plaques and NFT, associated with prominent synaptic defects and cognitive deficits. Hence, $\delta$-secretase facilitates AD pathogenesis independent of any patient-derived mutation.
\end{abstract}

\section{Introduction}

Alzheimer's disease (AD), an age-dependent neurodegenerative disorder with progressive cognitive impairments, is characterized by the accumulation of the extracellular $\beta$-amyloid peptide $(\mathrm{A} \beta)$ within the brain along with hyperphosphorylated and cleaved forms of the microtubuleassociated protein, Tau, as the pathological hallmarks. It is known that metabolic dysfunction of amyloid- $\beta$ precursor protein (APP) and abnormal intraneuronal Tau protein phosphorylation leads to the formation of senile plaques and neurofibrillary tangles (NFT), respectively. AD is a complex and heterogeneous disorder, and several factors

\footnotetext{
Correspondence: Liming Cheng (limingcheng@tongji.edu.cn) or Keqiang Ye (kye@emory.edu)

'Department of Pathology and Laboratory Medicine, Emory University School of Medicine, Atlanta, GA 30322, USA

${ }^{2}$ Division of Spine, Department of Orthopedics, Tongji Hospital affiliated to

Tongji University School of Medicine, Shanghai 200065, China

Full list of author information is available at the end of the article

Edited by P. G. Mastroberardino
}

play critical roles in its pathogenesis ${ }^{1}$. The defining pathologic features of amyloid plaques and NFTs are strongly intertwined with chronic neuroinflammation and extensive neuronal degenerative events ${ }^{2}$. Hippocampus, especially the CA1 region is responsible for learning and memory, which displays the main pathologies and responsible for dementia, the most critical symptoms in Alzheimer's disease ${ }^{3,4}$. Despite considerable research efforts in the field, the pathogenesis of $\mathrm{AD}$ remains obscure. Although numerous theories including an amyloid cascade hypothesis have been proposed ${ }^{5}$, none of the propositions temporally and spatially elucidates the $\mathrm{AD}$ pathology initiation and progression $^{6,7}$.

Mammalian asparaginyl endopeptidase (AEP, gene name: $L G M N$ ) is an endo-lysosomal cysteine protease that cleaves after asparagine residues. We show that AEP acts as $\delta$-secretase that proteolytically cleaves both APP and $\mathrm{Tau}$, and these proteins were fragmented by $\delta$-secretase in human AD brains ${ }^{8}$. Delta-secretase autocleavage and

\section{(c) The Author(s) 2020}

(c) (i) Open Access This article is licensed under a Creative Commons Attribution 4.0 International License, which permits use, sharing, adaptation, distribution and reproduction cc) in any medium or format, as long as you give appropriate credit to the original author(s) and the source, provide a link to the Creative Commons license, and indicate if changes were made. The images or other third party material in this article are included in the article's Creative Commons license, unless indicated otherwise in a credit line to the material. If material is not included in the article's Creative Commons license and your intended use is not permitted by statutory regulation or exceeds the permitted use, you will need to obtain permission directly from the copyright holder. To view a copy of this license, visit http://creativecommons.org/licenses/by/4.0/. 
activation is induced by $\mathrm{pH}$ reduction in the brain during aging. Interestingly, $\delta$-secretase expression levels and activity are escalated in aged mice and human $A D$ and $P D$ brains ${ }^{9,10}$. Most recently, we report that $C / E B P \beta$, an inflammatory cytokine-mediated transcription factor, regulates $\delta$-secretase expression in an age-dependent manner in AD and PD brains ${ }^{11,12} . \delta$-secretase cuts APP at both N373 and N585 residues in the ectodomain, and facilitates $A \beta$ production by decreasing the steric hindrance for $\beta$-amyloid site cleaving enzyme 1 (BACE1). The depletion of $\delta$-secretase significantly reduces $A \beta$ production and senile plaque formation in 5XFAD mouse brains, leading to the restoration of synaptic activity and cognitive functions ${ }^{10}$. Additionally, $\delta$-secretase cuts Tau at N255 and N368 residues and abolishes its microtubule assembly activity, resulting in its aggregation and NFT formation. Notably, the cleaved Tau 1-368 fragment is neurotoxic. The deletion of $\delta$-secretase from Tau P301 mice reverses the synaptic plasticity defect and cognitive dysfunctions ${ }^{9}$. Co-expression of $\delta$-secretase-truncated human APP C586-695 fragment and human Tau N368 truncate in the hippocampus of wild-type mice additively drives AD-like pathogenesis and cognitive dysfunctions ${ }^{13}$, suggesting that $\delta$-secretase activity may promote AD-like pathology onset when both APP and Tau are abundant. Recently, we report that SRPK2, a cell-cycle-regulated kinase, phosphorylates S226 residue on $\delta$-secretase and activates the enzyme, triggering its cytoplasmic translocation from the lysosomes ${ }^{14}$. On the contrary, BDNFmediated Akt phosphorylates T322 residue on $\delta$-secretase and inactivates it, sequestering its lysosomal residency ${ }^{15}$. Remarkably, Tau N368 fragments are demonstrable in the CSF and tissue lysate of human AD patients. Concentrations of Tau N368 in these CSF samples are both correlated tightly with Tau in PET images from AD brains and related to the extent of disease deterioration, indicating that $\delta$-secretase is proteolytically activated in $\mathrm{AD}$ patient brains and mediates Tau pathologies ${ }^{16}$, fitting with the observations that abnormal Tau hyperphosphorylation and deposition are better related to disease severity and cognitive disorders ${ }^{17}$. Taken together, these findings support the hypothesis that $\delta$-secretase plays a pivotal role in AD pathogenesis. In the present study, we established a new mouse AD model with both human wild-type APP and Tau (hAPP/hMAPT). Remarkably, overexpression of $\delta$-secretase instigates the formation of senile plaques and NFTs in these mice and incurs an extensive neuronal loss and prominent neuro-inflammation, leading to demonstrable cognitive dysfunctions.

\section{Results}

Characterization of hAPP/hMAPT double-transgenic mice

Currently, all of $\mathrm{AD}$ mouse models employ various human AD patient-derived APP mutants, or even combined with
Tau P301 mutants, in order to recapitulate the senile plaques and NFT pathologies. Recently, we report that coexpression of $\delta$-secretase-cleaved human APP C586-695 and Tau 1-368 fragments in the hippocampus of wild-type mice elicits demonstrable senile plaques and NFT, associated with evident neuronal loss and neuroinflammation, leading to cognitive disorders ${ }^{13}$. This finding suggests that $\delta$-secretase activity might be sufficient for initiating ADlike pathologies in the presence of abundant APP and Tau substrates. To test this possibility, we crossed the human wild-type APP transgenic I5 mice ${ }^{18}$ with human wild-type MAPT transgenic mice (htau) ${ }^{19}$ to obtain hAPP/hMAPT double-transgenic mice. Immunoblotting revealed that both human APP and human Tau are highly upregulated in double-transgenic mice, associated with C/EBP $\beta$ upregulation and $\delta$-secretase escalation, as compared to WT control, hAPP or hMAPT single transgenic mice (Fig. $1 \mathrm{~A}, 1^{\text {st }}-6^{\text {th }}$ panels). The upregulated $\delta$-secretase was proteolytically cleaved and active, accompanied by demonstrable APP N373 and Tau N368 in doubletransgenic mice, which were barely detectable in other mice. These two truncates were also demonstrable in the corresponding single transgenic mice. Accordingly, Tau was highly phosphorylated as revealed by the prominent AT8 signal in hAPP/hMAPT mice (Fig. 1A, $7^{\text {th }}$ $9^{\text {th }}$ panels). Immunofluorescent (IF) staining showed that APP C586 was augmented in double-transgenic mice, associated with palpable $A \beta$ activities. Moreover, Tau N368 and AT8 activities were increased in doubletransgenic mice. These events were consistent with elevated $\delta$-secretase levels. Further, expression of Iba1, a marker for microglial activation, was increased; by contrast, substantial neuronal loss and a pro-apoptotic trend were detected in hAPP/hMAPT double-transgenic mice, which was validated with enhanced active Caspase-3 expression (Fig. 1B). MAP2 staining in the remained neurons demonstrated a significant decrease in hMAPT single-transgenic and hAPP/hMAPT double-transgenic mice. In alignment with these observations, $A \beta$ and Thioflavin $\mathrm{S}(\mathrm{ThS})$ double staining $(\mathrm{A} \beta / \mathrm{ThS})$ showed that augmented $A \beta$ was aggregated into ThS-positive fibrils in the cortex of one-year-old double-transgenic mice. On the other hand, AT8/ThS co-staining indicated that detectable p-Tau in both hAPP and hMAPT single transgenic mice but only NFT stained with both AT8 and ThS were found in the cortex of one-year-old hAPP/hMAPT double-transgenic mice (Fig. 1C). Quantitative RT-PCR (qRT-PCR) showed that mouse APP mRNA levels remained comparable among these mouse strains. Mouse MAPT levels in hMAPT mice and hAPP/hMAPT mice were approximately half of those in WT or hAPP mice because endogenous mouse MAPT gene was knocked out in hMAPT mice (Supplementary Figure 1). Cognitive dysfunction was observed as assessed by Morris 


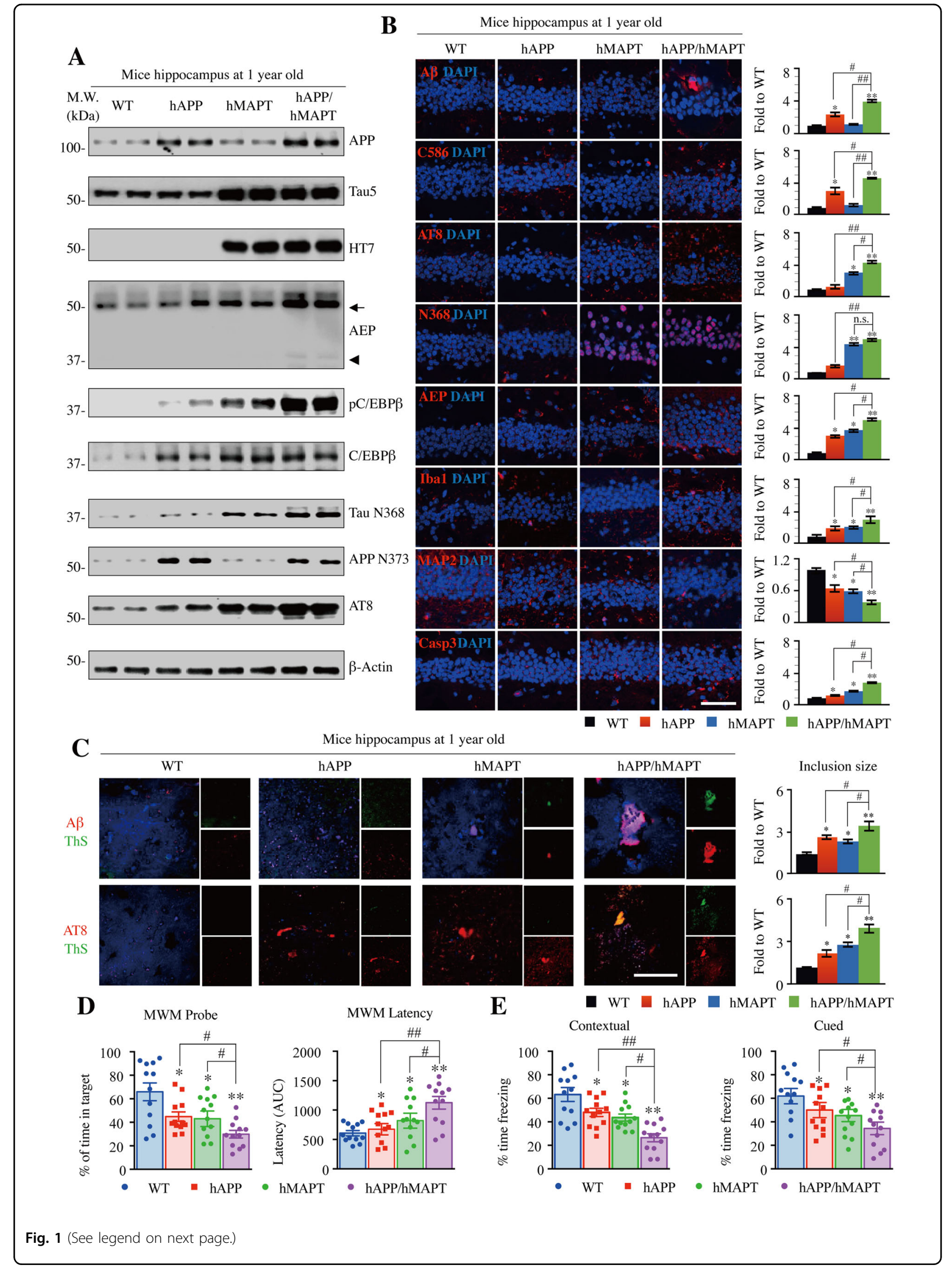


(see figure on previous page)

Fig. 1 Characterization of hAPP/hMAPT double-transgenic mice. A Western blot assays demonstrated AD-related protein levels in human wildtype APP and human wild-type Tau double-transgenic mice, a new AD mouse model. Wild-type mice, human wild-type APP transgenic mice, human wild-type Tau single transgenic mice, human wild-type APP, and Tau double-transgenic mouse hippocampus were collected at 12 months of age. APP NT (for both mouse and human APP), Tau5 (for both mouse and human Tau), HT7 (for human Tau) antibodies were applied to validate the double-transgenic model. Arrow indicated full-length AEP ( 56 kDa), arrowhead indicated activated (mature) AEP ( 37 kDa). Quantitative analysis of relative mRNA levels can be found Supplementary Figure 1. B Immunofluorescent staining showed AD-like pathologies in the WT, two single transgenic lines, and human APP/MAPT mice, including amyloid deposition (A $\beta$ antibody), Tau hyperphosphorylation (AT8 antibody),

neuroinflammation (Iba1 antibody) and the dendritic reduction in the remained neurons (MAP2) as well as cleaved-Caspase3 (Casp3). The specific antibodies of AEP-cleaved APP (C586) and Tau (N368) were used for the detection of AEP activation (left, sale bar: $50 \mu$ m). Quantitative analysis the percentage of positive cells. The number of Iba1+ microglia cell body was count and then calculated as mean $\pm \mathrm{SEM} \times 10^{3} / \mathrm{mm}^{3}$. (right, Mean $\pm \mathrm{SEM}$, $n=6$ mice for each group, one-way ANOVA with Dunnett's multiple-comparisons test. * as compared to WT mice, \# as comparison to hAPP/hMAPT mice). C The formation of amyloid plaques and neurofibrillary tangle (NFT) in four lines of mice at one-year old were shown by co-staining of ThS + $A \beta$ and ThS + AT8, respectively (left. Scale bar: $50 \mu \mathrm{m}$ ). Quantitative analysis to demonstrate the size of A + plaque and AT8 + NFT (right. Mean $\pm \mathrm{SEM}, n=6$ mice in each group, one-way ANOVA with Dunnett's multiple-comparisons test, ${ }^{*}$ as compared to WT mice, \# as comparison to hAPP/ hMAPT mice). D Morris water maze analysis as the percentage of time spent in the target quadrant in the probe trial (left) and the latency time (right, AUC: area under the curve) to the platform in the training days. (Mean $\pm \mathrm{SEM}, n=12$ mice for each group, one-way ANOVA with Dunnett's multiplecomparisons test, ${ }^{*}$ as compared to WT, \# as comparison to hAPP/hMAPT mice). E Fear conditioning tests. Contextual (left) and cued (right) fear conditioning were time-dependently impaired in mice after the virus. (Mean \pm SEM, $n=12$ mice for each group, one-way ANOVA with Dunnett's multiple-comparisons test, ${ }^{*}$ as compared to WT mice, \# as comparison to hAPP/hMAPT mice).

water maze and fear conditioning cognitive-behavioral tests when compared to age-matched wild-type mice. The impairments were the worst in hAPP/hMAPT doubletransgenic mice (Fig. 1D), consistent with the most prominent $\mathrm{AD}$-like pathologies in these double-transgenic animals. Hence, hAPP/hMAPT double-transgenic mice possess some of the AD-related pathologies that are absent in the single transgenic mice.

\section{Delta-secretase triggers AD-like pathologies and cognitive impairments in hAPP/hMAPT double-transgenic mice}

To determine whether overexpression of $\delta$-secretase is sufficient to trigger AD-like pathologies in hAPP/hMAPT double-transgenic mice, we injected the AAV9-AEP virus into the hippocampus of 2 month-old wild-types, hAPP, hMAPT and hAPP/hMAPT transgenic mice. Meanwhile, the AAV9-Control virus without overexpression of AEP was injected into the same location of 2 month-old wildtype and hAPP/hMAPT mice as the control for the effect of surgery and viral infection. There was no complication or abnormality after injection. No virus injection-caused AEP activation was observed in the AAV-Control group. In 3 months after injection, we monitored the expression of $\delta$-secretase and various biochemical events by immunoblotting. Overexpressed $\delta$-secretase was auto-cleaved and activated, which triggered APP cleavage into N373 and Tau into Tau N368 fragments, respectively, associated with robust Tau phosphorylation. Noticeably, expression of the inflammation- and $A \beta$-regulated transcription factor, $C / E B P \beta$, was escalated, and it was also highly phosphorylated in $\delta$-secretase-infected hAPP, hMAPT and the double-transgenic mice (Fig. 2A). IF analysis showed that overexpression of $\delta$-secretase increased APP C586, A $\beta$, Tau N368, and AT8 signals, but these activities were all further augmented in hAPP/ hMAPT double-transgenic mice, as were Iba1 immunoreactivity and neuronal loss. In hippocampus region, only intracellular $A \beta$ rather than extracellular $A \beta$ was found and then quantified (Fig. 2B). Surprisingly, extracellular insoluble $\mathrm{A} \beta$-positive plaque was detected in the cortex region. As demonstrated in Fig. $2 \mathrm{C}$, anti- $\mathrm{A} \beta / \mathrm{ThS}$ and anti-AT8/ThS co-staining indicated that overexpression of $\delta$-secretase increased double-positive signals in both hAPP and hMAPT single transgenic mice. The maximal effect occurred to hAPP/hMAPT doubletransgenic mice, which exhibited $A \beta$ aggregates that were ThS-positive, and AT8 inclusions that were also ThSpositive, respectively (Fig. 2C). Quantitative analysis for the inclusion sizes and the percentages of the ThS + area in both $\mathrm{A} \beta+$ plaque and AT8 + NFT demonstrated that formations of amyloid plaque and NFT were accelerated in the double transgenic mice with overexpression of AEP (Fig. 2D). Thus, overexpression of $\delta$-secretase strongly increases both APP and Tau cleavage, resulting in senile plaque deposition and NFT formation, which are associated with neuroinflammation and neuronal loss.

Fitting with immunoblotting findings, $\delta$-secretase enzymatic activity was the highest in the doubletransgenic mice, relative to the other three mouse strains. Injection of AAV-AEP further escalated its enzymatic activities (Fig. 3A). Quantitative analysis of inflammatory cytokines including IL-1 $\beta$, IL-6, and TNF $\alpha$ disclosed the same pattern. All were more abundant in double-transgenic mice after $\delta$-secretase injection (Fig. $3 \mathrm{~B}-\mathrm{D})$. A $\beta$ ELISA showed that the most copious human A $\beta$ 1-40 and 1-42 were found in hAPP/hMAPT mice, followed by hAPP transgenic mice (Fig. 3E, F). Levels of mouse $A \beta 1-40$ or 1-42 concentrations also were the 


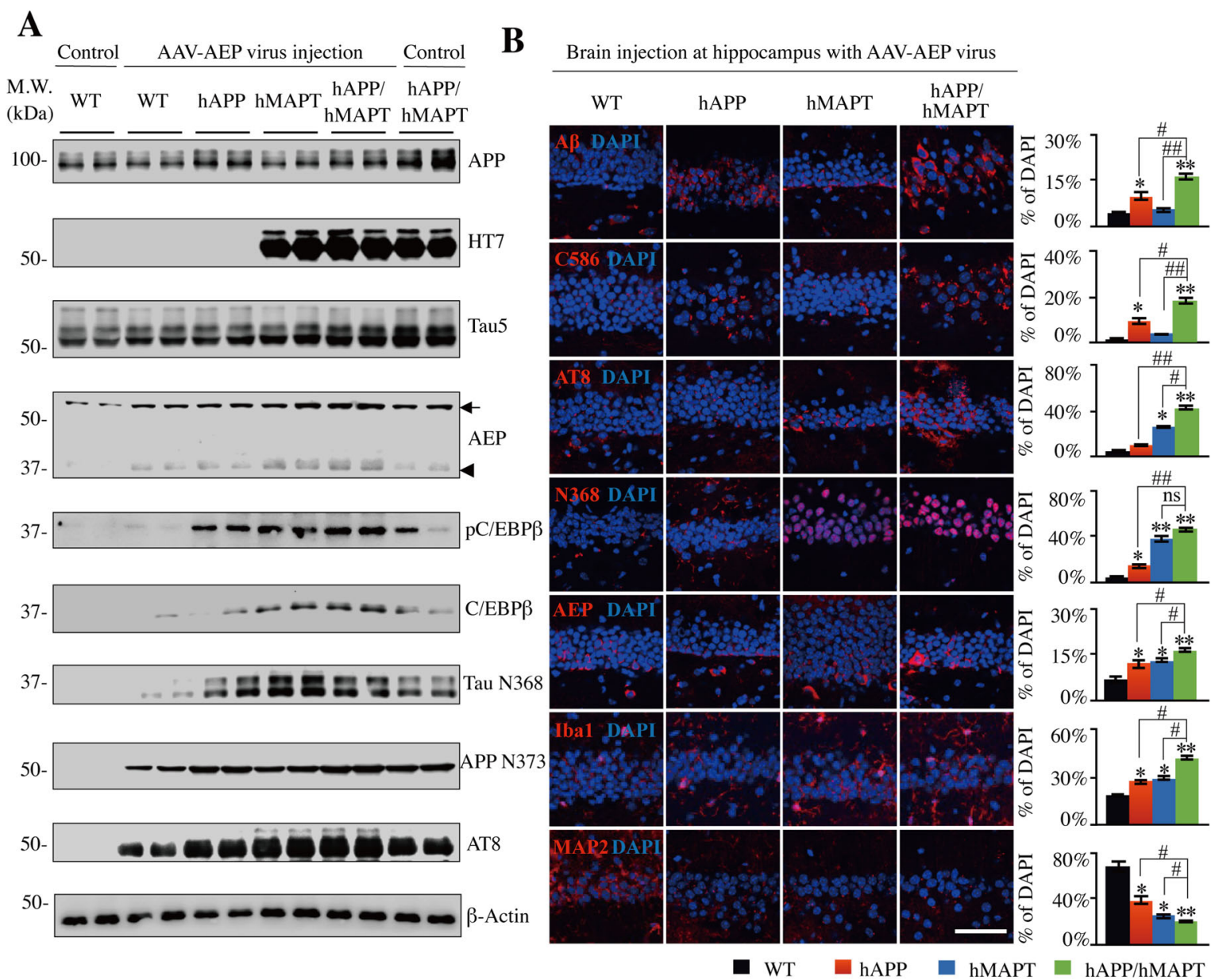

C Brain injection at hippocampus with AAV-AEP virus
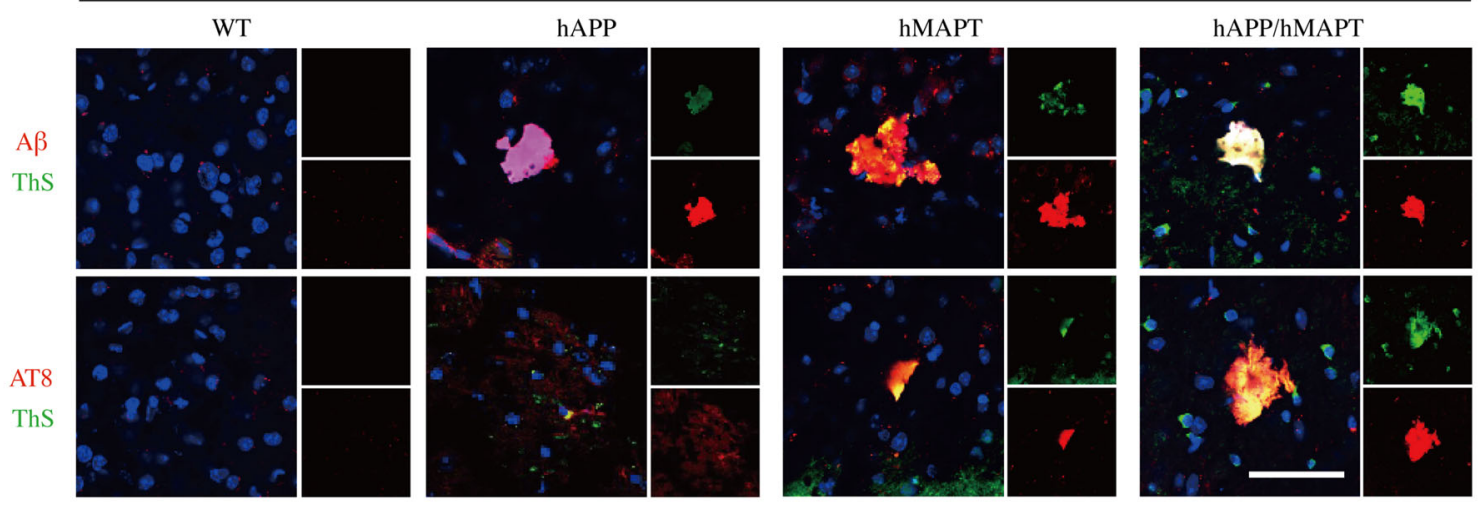

D
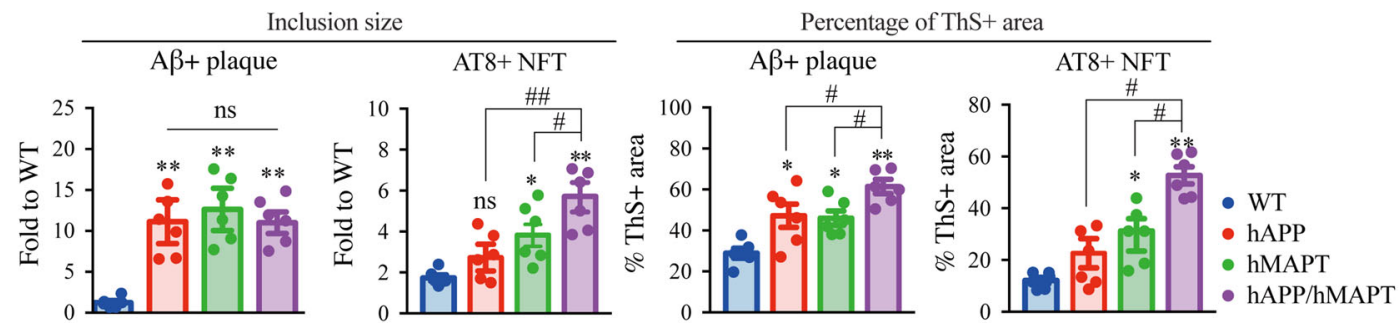

Fig. 2 (See legend on next page.) 
(see figure on previous page)

Fig. 2 Delta-secretase enhances AD-like pathologies in hAPP/hMAPT double-transgenic mice. A $\delta$-secretase overexpression induces APP and Tau proteolytic cleavage in the double-transgenic mice. Western blot assays demonstrated $\delta$-secretase-cleaved APP, and Tau fragments promoted AD-like pathologies in human wild-type APP and Tau double-transgenic mice. Arrow indicated full-length AEP ( 56 kDa), arrowhead indicated activated (mature) AEP ( $37 \mathrm{kDa})$. WT, hAPP, hMAPT, hAPP/hMAPT mice at the age of 2-3 months old were injected with AAV-AEP virus in the bilateral hippocampus for 3 months. WT mice (lane 1 and 2) and hAPP/hMAPT mice (lane 11 and 12) with AAV-Control virus injection were compared. APP NT (for both mouse and human APP), Tau5 (for both mouse and human Tau), HT7 (for human Tau) antibodies were applied to validate the double-transgenic model. AEP (6E3) antibodies were used to verify the efficiency of AAV-AEP virus injection. B 8 -secretase elicits AD-like pathologies in hAPP/hMAPT mice. Immunofluorescent staining showed AD-like pathologies in the WT, hAPP, hMAPT, and hAPP/MAPT mice injected with AAV-AEP virus, including amyloid deposition (A $\beta$ antibody), Tau hyperphosphorylation (AT8 antibody), neuroinflammation (Iba1 antibody), and the dendritic reduction in the remained neurons (MAP2). The specific antibodies of AEP-cleaved APP (C586) and Tau (N368) were used for the detection of AEP activation (left, scale bar: $50 \mu \mathrm{m}$ ). Quantitative analysis the percentage of positive cells. The number of Iba1+ microglia cell bodies was count and then calculated as mean \pm SEM $\times 10^{3} / \mathrm{mm}^{3}$. (right, Mean $\pm \mathrm{SEM}, n=6$ mice for each group, one-way ANOVA with Dunnett's multiplecomparisons test. ${ }^{*}$ as compared to WT mice, \# as comparison to hAPP/hMAPT mice). C The formation of amyloid plaques and neurofibrillary tangle (NFT) in four lines of mice treated with the AAV-AEP virus was shown by co-staining of ThS $+A \beta$ and ThS + AT8, respectively. Scale bar: $50 \mu m$. $\mathbf{D}$ Quantitative analysis to demonstrate the size of A $\beta$ + plaque and AT8 + NFT (left, Mean \pm SEM, $n=6$ in each group, one-way ANOVA with Dunnett's multiple-comparisons test, ${ }^{*}$ as compared to WT + AAV-AEP virus, \# as comparison to hAPP/hMAPT + AAV-AEP virus) and the proportion (\%) of Thioflavin S positive area (right). (Mean \pm SEM, $n=6$ in each group, one-way ANOVA with Dunnett's multiple-comparisons test, ${ }^{*}$ as compared to WT + AAV-AEP virus, \# as comparison to hAPP/hMAPT + AAV-AEP virus).

highest in hAPP/hMAPT mice after AAV-AEP injection (Fig. 3G, H). The absolute values of human $A \beta 40$ and A 342 in hAPP mice were half of the ones in human wildtype APP mice (hAPPwt I5) mice strain at a similar age (the molecular weights of human $A \beta 40$ and $A \beta 42$ were $4514 \mathrm{~g} / \mathrm{mol}$ and $4330 \mathrm{~g} / \mathrm{mol}$, respectively), demonstrating its heterozygous genetic background of human APP genes $^{18}$. Even though, mouse $A \beta 40$ and $A \beta 42$ levels of hAPP mice were comparable to WT and hMAPT, indicating the neurotoxicity of exogenous human $A \beta$ associated with the elevated AEP resulted in the accumulation of intrinsic mouse $A \beta$. Immunohistochemical (IHC) staining with anti-A $\beta$ validated these observations (Fig. 3I, upper panels). AT8 staining showed p-Tau activities were the strongest in double-transgenic mice, which were further elevated upon $\delta$-secretase overexpression (Fig. 3I, lower panels).

Electron microscopic (EM) analysis indicated that apparent synapse loss was the greatest in double-transgenic mice, which was gradually reduced from hMAPT to hAPP to WT mice. Overexpression of $\delta$-secretase stimulated the reduction of synapses. The fewest synapses were found in hAPP/hMAPT mice after $\delta$-secretase overexpression (Fig. 4A). A similar pattern was observed in dendritic spines in these mice by Golgi staining (Fig. 4B). As above, overexpression of $\delta$-secretase greatly reduced the dendritic spines in hAPP/hMAPT mice. In alignment with these observations, Gallyas silver staining revealed that the pathological aggregation increased in hAPP/hMAPT double-transgenic mice (Fig. 4C, upper panels), fitting with the findings by anti-A $\beta /$ ThS and AT8/ThS co-staining in Fig. 2C. Overexpression of $\delta$-secretase substantially enhanced this effect in both hAPP and hMAPT single transgenic mice with the climax effect that occurred to hAPP/hMAPT mice (Fig. 4C, lower panels). The behavioral tests showed that $\delta$-secretase elicited significant cognitive impairments in hAPP/hMAPT mice compared to the control virus, consistent with the prominent AD-like pathologies in these mice (Fig. 4D, E). Collectively, these studies demonstrate that overexpression of $\delta$-secretase elicits both APP and Tau cleavage and leads to AD-like pathologies and cognitive impairments in hAPP/hMAPT double-transgenic mice regardless of APP or Tau mutation.

\section{Methods \\ Animals}

Wild-type C57BL/6 J, hAPP (hAPPwt line I5), and hMAPT (htau) mice were purchased from the Jackson Laboratory (MMRRC stock \# 000664, \#004662, \#005491, respectively). Human wild-type APP/MAPT doubletransgenic mice (hAPP/hMAPT) were generated by crossing hAPP (hAPPwt line I5) and hMAPT (htau) mice. Human MAPT genes in both hMAPT and hAPP/hMAPT mice are heterozygous $( \pm)$ since the homozygous insert is embryonic lethal ${ }^{19}$. Animal care and handling were performed according to the Declaration of Helsinki and Emory Medical School guidelines. Both male and female mice were used in the experiments. Mice were randomized into different groups by using a random number table. Investigators were blinded to the group allocation during the animal experiments.

\section{AAV vector packaging and stereotaxic injection}

The AAV9 particles encoding AEP with CMV promoter were prepared by Viral Vector Core at Emory University. The AAV9 backbone plasmid without AEP cDNA was applied as the control for the effect of surgery and viral infection. Bilateral intracerebral injection of AAV-Control or AAV-AEP was performed stereotactically at coordinates posterior $1.94 \mathrm{~mm}$, lateral $1.4 \mathrm{~mm}$, ventral $2.2 \mathrm{~mm}$ 
A

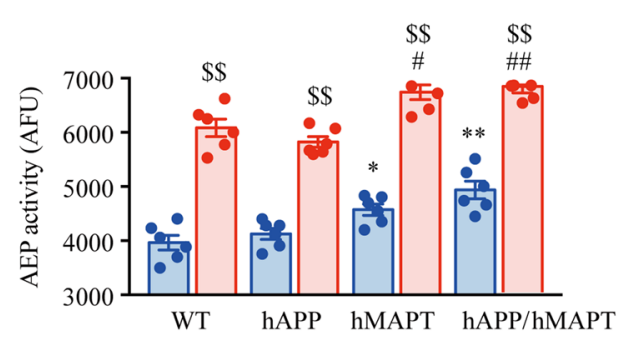

C

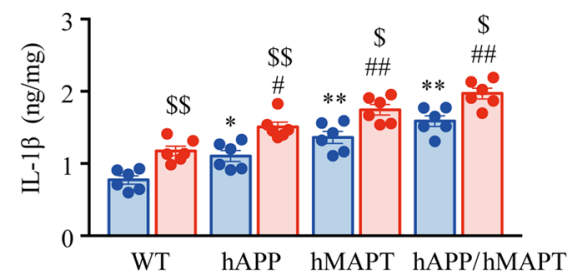

E

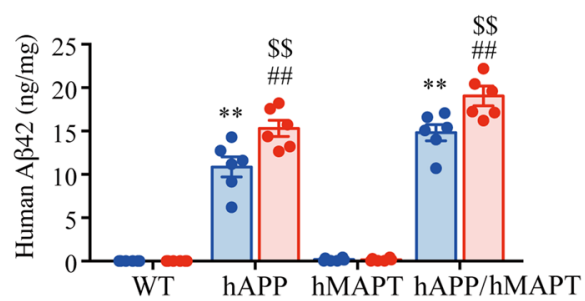

G

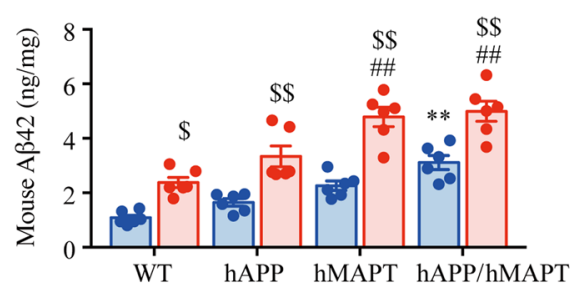

I
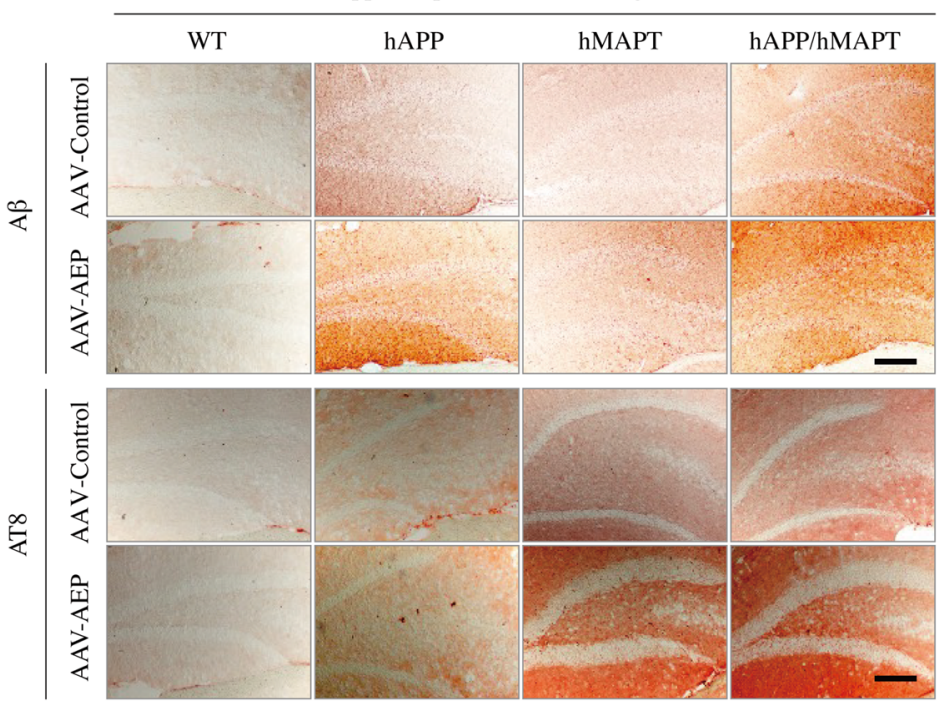

B

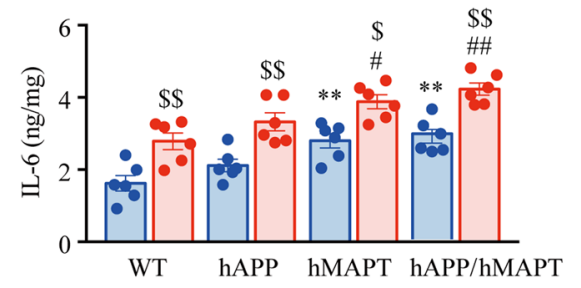

D

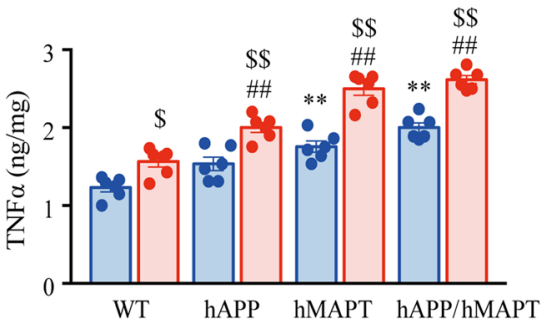

F

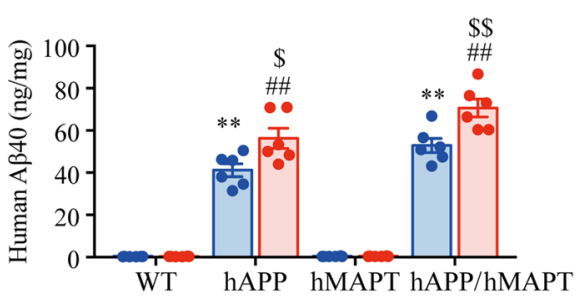

H

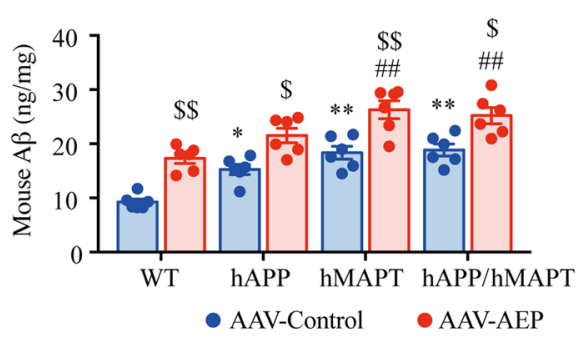


(see figure on previous page)

Fig. 3 Overexpression $\delta$-secretase in the hippocampus of hAPP/hMAPT mice induces $A \beta$ escalation and neuroinflammation. $A$-secretase enzymatic assay. AEP enzymatic activity assay showed AEP activity in all the four lines of mice injected with AAV-AEP and AAV-Control virus. $\delta$ secretase was increased in the hAPP/hMAPT mice, and overexpression of $\delta$-secretase displayed the highest activities in hAPP/hMAPT doubletransgenic mice (Mean \pm SEM, $n=6$ mice for each group, two-way ANOVA with Bonferroni multiple-comparisons test. * as compared to the WT mice + AAV-Control Virus; \# as compared to the WT mice + AAV-AEP virus; $\$$ as compared to AAV-Control virus in same mouse strain). B ELISA analysis of neuroinflammation factors IL-6 in the brain lysates from four lines of mice treated with AAV-AEP and AAV-Control virus (Mean \pm SEM, $n=6$ mice for each group, two-way ANOVA with Bonferroni multiple-comparisons test. ${ }^{*}$ as compared to WT mice + AAV-Control virus; \# as compared to the WT mice + AAV-AEP virus; $\$$ as compared to AAV-Control virus in same mouse strain). C ELISA analysis of neuroinflammation factors IL-1 $\beta$ in the brain lysates from four lines of mice treated with AAV-AEP and AAV-Control virus (Mean $\pm S E M, n=6$ mice for each group, two-way ANOVA with Bonferroni multiple-comparisons test. ${ }^{*}$ as compared to WT mice + AAV-Control virus; \# as compared to the WT mice + AAV-AEP virus; $\$$ as compared to AAV-Control virus in same mouse strain). D ELISA analysis of neuroinflammation factors TNFa in the brain lysates from four lines of mice treated with AAV-AEP and AAV-Control virus (Mean $\pm \mathrm{SEM}, n=6$ mice for each group, two-way ANOVA with Bonferroni multiple-comparisons test. * as compared to WT mice + AAV-Control virus; \# as compared to the WT mice + AAV-AEP virus; $\$$ as compared to AAV-Control virus in same mouse strain). E ELISA analysis of human A 342 in the brain lysates from four lines of mice treated with AAV-AEP and AAV-Control virus. (Mean \pm SEM, $n=6$ mice for each group, two-way ANOVA with Bonferroni multiple-comparisons test. * as compared to WT mice + AAV-Control virus; \# as compared to the WT mice + AAV-AEP virus; \$ as compared to AAV-Control virus in same mouse strain). $\mathbf{F}$ ELISA analysis of human A 40 in the brain lysates from four lines of mice treated with AAV-AEP and AAV-Control virus. (Mean $\pm S E M, n=6$ mice for each group, two-way ANOVA with Bonferroni multiplecomparisons test. * as compared to WT mice + AAV-Control virus; \# as compared to the WT mice + AAV-AEP virus; \$ as compared to AAV-Control virus in same mouse strain). G ELISA analysis of mouse AB42 in the brain lysates from four lines of mice treated with AAV-AEP and AAV-Control virus. (Mean $\pm \mathrm{SEM}, n=6$ mice for each group, two-way ANOVA with Bonferroni multiple-comparisons test. ${ }^{*}$ as compared to WT mice + AAV-Control virus; \# as compared to the WT mice + AAV-AEP virus; $\$$ as compared to AAV-Control virus in same mouse strain). $\mathbf{H}$ ELISA analysis of mouse Aß40 in the brain lysates from four lines of mice treated with AAV-AEP and AAV-Control virus. (Mean $\pm S E M, n=6$ mice for each group, two-way ANOVA with Bonferroni multiple-comparisons test. * as compared to WT mice + AAV-Control virus; \# as compared to the WT mice + AAV-AEP virus; $\$$ as compared to AAV-Control virus in same mouse strain). (I) Immunohistochemistry characterization of A $\beta$ and AT8. The hippocampal sections of the indicated mice infected with AAV-AEP and AAV-Control virus were stained with anti-A $\beta$ or AT8. Overexpression of $\delta$-secretase increased A 3 and $p$ Tau signals in the double-transgenic mice (left, scale bar: $30 \mu \mathrm{m}$ ). Quantitative analysis of the immunohistochemical signals in each group (right, Mean \pm SEM, $n=6$ mice for each group, two-way ANOVA with Bonferroni multiple-comparisons test. * as compared to WT mice + AAV-Control virus).

relative to Bregma. $2 \mu$ l of viral suspension containing $2 \times$ $10^{11}$ vector genome $(\mathrm{vg})$ was injected into each point using $10 \mu \mathrm{l}$ glass syringes with a fixed needle.

\section{Immunoblotting analysis}

Mouse brain tissue samples were lysed in lysis buffer (50 mM Tris, pH 7.4, $40 \mathrm{mM} \mathrm{NaCl}, 1 \mathrm{mM}$ EDTA, $0.5 \%$ Triton X-100, $1.5 \mathrm{mM} \mathrm{Na} \mathrm{VO}_{4}, 50 \mathrm{mM} \mathrm{NaF}, 10 \mathrm{mM}$ sodium pyrophosphate, $10 \mathrm{mM}$ sodium-glycerophosphate, supplemented with protease inhibitors cocktail) on ice for $30 \mathrm{~min}$. The lysates were centrifuged for 10 minutes at $15,000 \mathrm{rpm}$. Protein concentration from the supernatant was determined using a Coomassie Brilliant Blue protein assay kit (Bio-Rad). The same amount of the supernatant was boiled in SDS loading buffer. After SDSPAGE, the samples were transferred to a nitrocellulose membrane. The membranes were blocked in 5\% non-fat milk for 1 hour at room temperature and then incubated with primary antibody at $4{ }^{\circ} \mathrm{C}$ overnight. Primary antibodies to the following targets were used: APP NT (Millipore, \# MAB348), HT7 (Invitrogen, \# MN1000), Tau5 (Millipore, \# MAB361), C/EBP $\beta$ C19 (Santa Cruz, \# sc150), p-C/EBP $\beta$ T188/T235 (CST, \# 3084), AEP (11B7 as a gift from Dr. Colin Watts), AT8 (Thermo, \#MN1020) and $\beta$-Actin (Sigma-Aldrich, \# A1978). Both Tau N368 and APP C586 antibodies were generated in our lab and characterized in our previous publications ${ }^{9,10}$. Then the blots were incubated with IRDye $800 \mathrm{CW}$-conjugated affinity-purified anti-mouse or anti-rabbit IgG secondary antibody (Rockland). Immunoreactive bands were visualized using the Odyssey Infrared Imaging System (Licor Biosciences, Lincoln, NE, USA). ImageJ software was applied to quantify the immunoblotting intensity.

\section{Immunostaining and Thioflavin S staining}

The primary antibodies used were A $\beta$ (Sigma, \# A8354), AEP (11B7 as a gift from Dr. Colin Watts), Tau N368, APP C586, Iba1 (Sako, \#NCNP24), Map2 (Sigma, \# AB15452), AT8 (Thermo, \#MN1020) and cleaved-Caspase3 (CST, \#9664). Immunochemistry staining was performed with the immunohistochemistry staining kit (Invitrogen). Briefly, 4\% paraformaldehyde (PFA)-fixed mice brain frozen slides were treated with $3 \%$ hydrogen peroxide at room temperature for 10 minutes. Manufacturer-supplied blocking buffer (Invitrogen) was used for each reaction. Then the sections were incubated with primary antibodies overnight at $4{ }^{\circ} \mathrm{C}$. Biotinconjugated secondary antibodies (Jackson ImmunoResearch), streptavidin-conjugated HRP (Invitrogen) were applied to enhance the signals. For immunofluorescence staining, brain sections were incubated with an Alexa Fluor 594 conjugated isotype-specific secondary antibody (Jackson ImmunoResearch, West Grove, PA) for 1 hour at room temperature. To quantify and compare $A \beta$ in CA 1 region, intracellular $A \beta$ was measured and $A \beta$-positive cells were 
A

Mice hippocampus electron microscope exam

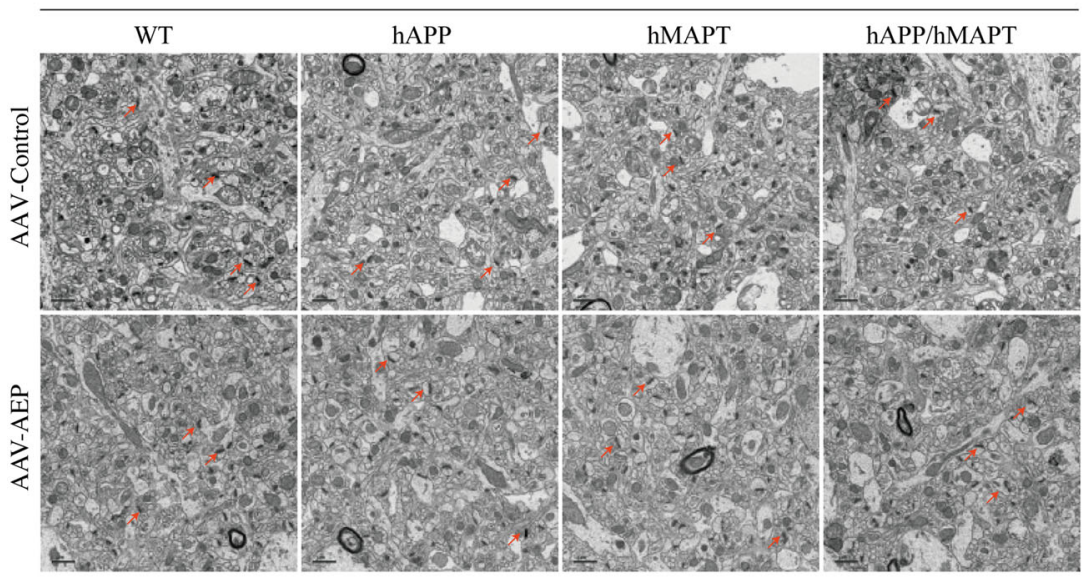

Synapses density in hippocampus

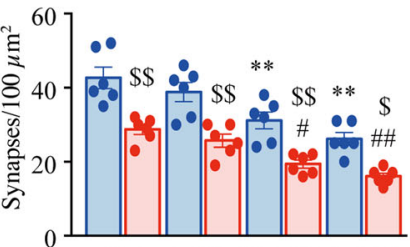

WT hAPP hMAPT hAPP/

hMAPT

- AAV-Control AAV-AEP

B
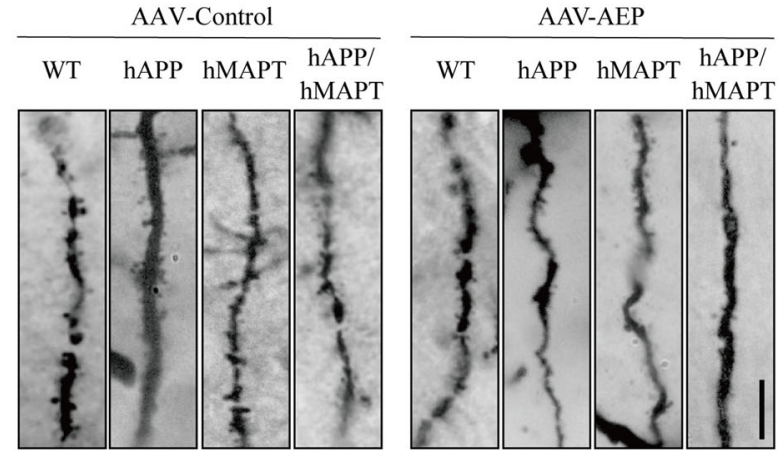

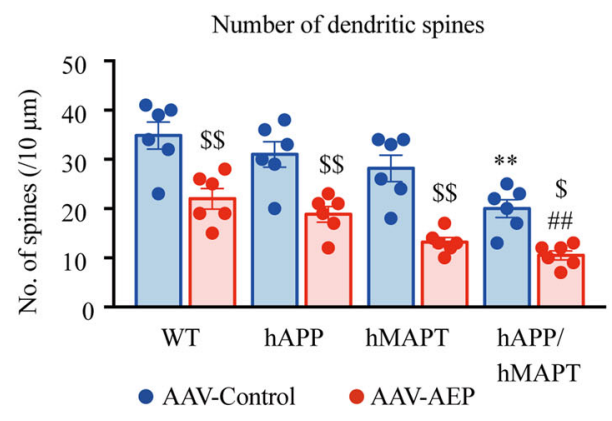

C

Mice hippocampus Gallyas silver staining

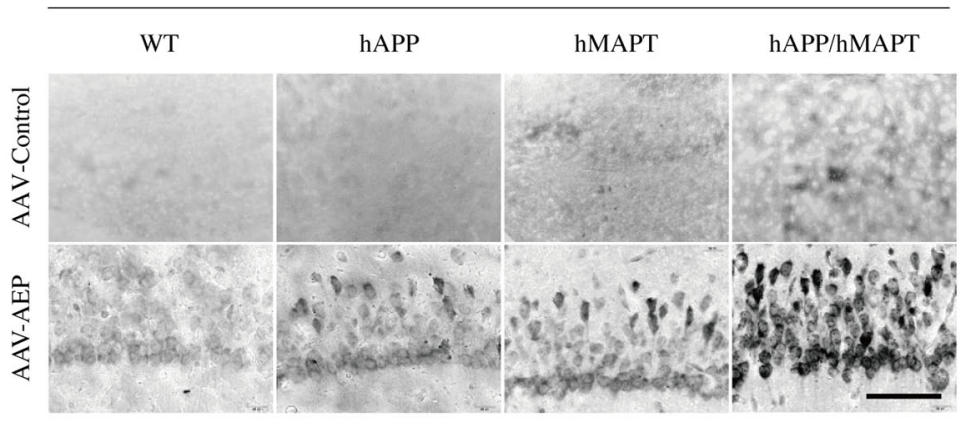

D

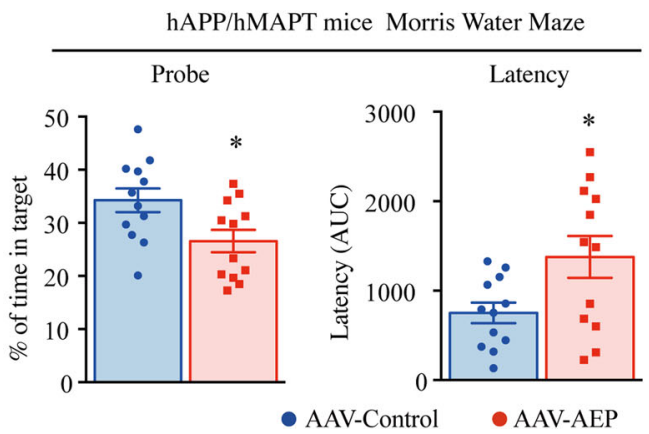

$\mathbf{E}$

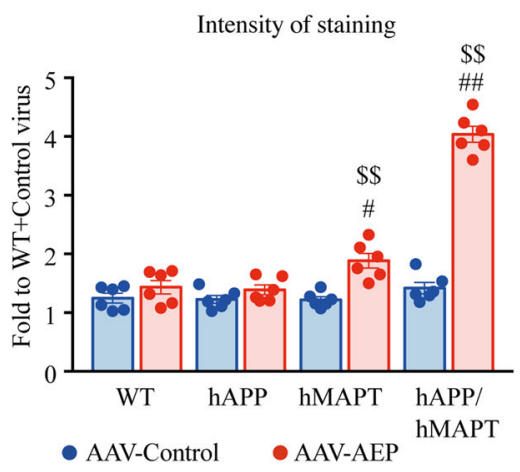

Fig. 4 (See legend on next page.) 
(see figure on previous page)

Fig. $4 \delta$-secretase overexpression triggers cognitive dysfunctions, associated with synapse and dendritic spine loss in hAPP/hMAPT mice. A Representative electron microscopy images of the synaptic structures at the CA1 region at 3 months after the AAV-AEP virus injection. Arrows indicated the synapses (left, scale bar: $2 \mu \mathrm{m}$ ). Quantitative analysis of the synaptic density in each group (right, Mean \pm SEM, $n=6$ mice for each group, two-way ANOVA with Bonferroni multiple-comparisons test. * as compared to WT mice + AAV-Control virus; \# as compared to the WT mice + AAVAEP virus; \$ as compared to AAV-Control virus in same mouse strain). B Golgi staining showed the dendritic spines from the apical dendritic layer of the CA1 region. (Left, Scale bar: $5 \mu \mathrm{m}$ ). Quantitative analysis of the spine density. (Right, Mean \pm SEM, $n=6$ mice for each group, two-way ANOVA with Bonferroni multiple-comparisons test. ${ }^{*}$ as compared to WT mice + AAV-Control virus; \# as compared to the WT mice + AAV-AEP virus; \$ as compared to AAV-Control virus in same mouse strain). C Gallyas silver staining for senile plaque and NFT in the hippocampus (Left. Scale bar: $50 \mu \mathrm{m})$. Quantitative analysis of the percentage of signal intensity (right, Mean \pm SEM, $n=6$ mice for each group, two-way ANOVA with Bonferroni multiplecomparisons test. * as compared to WT mice + AAV-Control virus; \# as compared to the WT mice + AAV-AEP virus; $\$$ as compared to AAV-Control virus in same mouse strain). D Morris water maze analysis as the percentage of time spent in the target quadrant in the probe trial (left) and the latency time (right, AUC: area under the curve) to the platform in the training days. (Mean \pm SEM, $n=12$ mice for each group, unpaired t-test, twotailed, * as compared to hAPP/hMAPT mice + AAV-Control virus). E Fear conditioning tests. Contextual (left) and cued (right) fear conditioning were time-dependently impaired in mice after the virus injection. (Mean $\pm \mathrm{SEM}, n=12$ mice for each group, unpaired t-test, two-tailed, ${ }^{*}$ as compared to hAPP/hMAPT mice + AAV-Control virus).

counted. For Thioflavin $S(T h S)+A \beta / A T 8$ double staining, slides were rinsed in PBS after finishing the A $\beta / A T 8$ first antibodies and the second antibody staining. Freshly dissolved $3.125 \mathrm{mg}$ Thioflavin $\mathrm{S}$ in $100 \mathrm{ml} 50 \%$ Ethanol. Incubated the A $\beta / A T 8$ stained slides in the ThS solution at room temperature for 7 minutes in the dark. Then wash the slides in 1\% Triton-X-100 and then PBS three times to exclude the signal of soluble plaque and debris. The fluorescence staining was visualized with an Olympus confocal microscope. Immunostaining images and their colocalization were quantified using Volocity 6.3 from Perkin Elmer and Fiji/ImageJ Coloc 2, respectively. The quantitative analysis methods of $\mathrm{ThS}$ staining were applied as previously reported ${ }^{20}$.

\section{AEP activity assay}

Tissue homogenates or cell lysates $(50 \mu \mathrm{g})$ were incubated in $100 \mu \mathrm{L}$ reaction buffer $(20 \mathrm{mM}$ citric acid, $60 \mathrm{mM}$ $\mathrm{Na}_{2} \mathrm{HPO}_{4}, 1 \mathrm{mM}$ EDTA, 0.1\% CHAPS, and $1 \mathrm{mM}$ DTT, $\mathrm{pH}$ 5.5) containing $10 \mu \mathrm{M}$ AEP substrate Z-Ala-Ala-AsnAMC (Bachem). AMC released by substrate cleavage was quantified by measuring at $460 \mathrm{~nm}$ in a fluorescence plate reader at $37^{\circ} \mathrm{C}$ in kinetic mode.

\section{ELISA quantitative analysis of $A \beta$}

To detect the concentration of $A \beta$ in total brain lysates, the mouse brains were homogenized in $8 \times$ mass of $5 \mathrm{M}$ guanidine $\mathrm{HCl} / 50 \mathrm{mM}$ Tris- $\mathrm{HCl}(\mathrm{pH} 8.0)$ and incubated at room temperature for $3 \mathrm{~h}$. Then the samples were diluted with cold reaction buffer (PBS with 5\% BSA and 0.03\% Tween-20, supplemented with protease inhibitor cocktail), and centrifuged at $16,000 \mathrm{~g}$ for $20 \mathrm{~min}$ at $4{ }^{\circ} \mathrm{C}$. The $\mathrm{A} \beta$ in the total brain was analyzed with human A 442 (KHB3441, Invitrogen), human $A \beta 40$ (KHB3481, Invitrogen), mouse A $\beta 42$ (KMB3441, Invitrogen), and mouse A $\beta 40$ (KMB3481, Invitrogen) ELISA kits according to the manufacturer's instructions. The $A \beta$ concentrations were determined by comparison with the standard curve.

\section{Real-time PCR}

Levels of mRNA were analyzed by real-time, quantitative PCR. RNA was isolated by Trizol (Life Technologies). Reverse transcription was performed with SuperScriptIII reverse transcriptase (Life Technologies). Gene-specific primers and probes were designed and bought from Taqman (Life Technologies). All real-time PCR reactions were performed using the ABI 7500-Fast Real-Time PCR System and TaqMan Universal Master Mix Kit (Life Technologies). The relative quantitative analysis of gene expression was calculated as the $2-\Delta \Delta \mathrm{Ct}$ method. For each data point, at least duplicated wells were used. GAPDH was used for housekeeping gene control. And each experiment was repeated at least 3 times.

\section{Golgi staining}

Mouse brains were fixed in $10 \%$ formalin for $24 \mathrm{~h}$ and then immersed in 3\% potassium dichromate for 3 days in the dark. The solution was changed each day. Then the brains were transferred into $2 \%$ silver nitrate solution and incubated for $24 \mathrm{~h}$ in the dark. Vibratome sections were cut at $60 \mu \mathrm{m}$, air-dried for $10 \mathrm{~min}$, dehydrated through 95 and $100 \%$ ethanol, cleared in xylene and cover-slipped. Bright-field images of pyramidal neurons in the hippocampus and cortex were taken at $100 \times$ magnification using a Zeiss Axioplan (Zeiss, Decatur, GA, USA) microscope. To measure the spine density, all clearly evaluable areas of $50-100 \mu \mathrm{m}$ of the secondary dendrites from each imaged neuron were used.

\section{Electron microscopy}

Synaptic density was determined by electron microscopy. After deep anesthesia, mice were perfused transcardially with $2 \%$ glutaraldehyde and $3 \%$ paraformaldehyde in PBS. Hippocampal slices were post-fixed in cold 1\% $\mathrm{OsO} 4$ for $1 \mathrm{~h}$. Samples were prepared and examined using standard procedures. Ultrathin sections $(90 \mathrm{~nm})$ were stained with uranyl acetate and lead acetate and viewed at 
$100 \mathrm{kV}$ in a JEOL 200CX electron microscope. Synapses were identified by the presence of synaptic vesicles and postsynaptic densities.

\section{Morris Water Maze}

Mice were trained in a round, water-filled tub (52-inch diameter) in an environment rich with extra-maze cues. An invisible escape platform was located in a fixed spatial location $1 \mathrm{~cm}$ below the water surface independent of subjects start position on a particular trial. In this manner, the subjects needed to utilize extra-maze cues to determine the platform's location. At the beginning of each trial, the mouse was placed in the water maze with their paws touching the wall from 1 of 4 different starting positions (N, S, E, W). Each subject was given 4 trials/day for 5 consecutive days with a 15 -min inter-trial interval. The maximum trial length was $60 \mathrm{~s}$, and if subjects did not reach the platform in the allotted time, they were manually guided to it. Upon reaching the invisible escape platform, subjects were left on it for an additional $5 \mathrm{~s}$ to allow for the survey of the spatial cues in the environment to guide future navigation to the platform. After each trial, subjects were dried and kept in a dry plastic holding cage filled with paper towels to allow the subjects to dry off. The temperature of the water was monitored every hour so that mice were tested in water that was between 22 and $25^{\circ} \mathrm{C}$. Following the 5 days of task acquisition, a probe trial was presented during which time the platform was removed and the percentage of time spent in the quadrant which previously contained the escape platform during task acquisition was measured over $60 \mathrm{~s}$. All latency trials were analyzed with MazeScan (Clever Sys, Inc.).

\section{Fear conditional tests}

The ability to form and retain an association between an aversive experience and environmental cues was tested with a standard fear conditioning paradigm that occurs for 3 days. Mice were placed in the fear conditioning apparatus (7" W, 7" D $312^{\prime \prime} \mathrm{H}$, Coulbourn) composed of Plexiglass with a metal shock grid floor and allowed to explore the enclosure for $3 \mathrm{~min}$. Following this habituation period, 3 conditioned stimuli (CS)-unconditioned stimulus (US) pairings were presented with a $1 \mathrm{~min}$ intertrial interval. The CS was composed of a $20 \mathrm{~s}, 85-\mathrm{dB}$ tone, and the US was composed of $2 \mathrm{~s}$ of a $0.5-\mathrm{mA}$ footshock, which was co-terminate with each CS presentation. One minute following the last CS-US presentation, mice were returned to their home cage. On day 2, the mice were presented with a context test, during which subjects were placed in the same chamber used during conditioning on day 1 , and the amount of freezing was recorded via a camera and the software provided by Coulbourn. No shocks were given during the context test. On day 3, a tone test was presented, during which time subjects were exposed to the CS in a novel compartment. Initially, animals were allowed to explore the novel context for $2 \mathrm{~min}$. Then the $85-\mathrm{db}$ tone was presented for $6 \mathrm{~min}$, and the amount of freezing behavior was recorded.

\section{Experimental design and statistical analysis}

All experiments were designed as described previously ${ }^{21}$ to provide sufficient power $(80-90 \%)$ to discriminate significant differences $(\alpha=0.05)$ in mean \pm standard error of the mean (SEM) between independent controls and experimental subjects. The numbers of tested subjects (n) were indicated in each figure legends. Statistical evaluations were performed with Student's t-test, one-way or two-way ANOVA, as indicated in figure legends. PostANOVA comparisons were made using the Dunnett's (one-way ANOVA) and Bonferroni (two-way ANOVA) correction. $p$ values $<0.05$ (indicated as * \#, \$) and $<0.01$ (indicated as ${ }^{* * *}, \#$ \#, \$) were considered as statistically significant, ns indicates not significant.

\section{Discussion}

In the current study, we show that viral expression of $\delta$ secretase elicits AD-like pathologies and cognitive disorders in hAPP/hMAPT double-transgenic mice. It is worth noting that hAPP/hMAPT mice possess no mutation in the human APP or MAPT gene. Strikingly, overexpressed $\delta$-secretase potently enhances $A \beta$ production and senile plaques deposits, accompanied by robust APP N373 fragmentation in both hAPP and hAPP/hMAPT transgenic mice, though APP N373 appeared less abundant in double transgenic line compared to hAPP mice (Fig. 1A), which might be due to its further proteolytically cleavage into even smaller fragment by other strongly activated proteases. Moreover, it also leads to the formation of prominent NFT in hMAPT and hAPP/hMAPT mice, accompanied by potent Tau N368 truncation. Accordingly, hippocampal neuronal cell death was manifested in $\delta$-secretase-injected hAPP/hMAPT doubletransgenic mice. On the other hand, among the tested strains of mice, neuro-inflammation upon $\delta$-secretase expression was the greatest in hAPP/hMAPT doubletransgenic mice, as was the cognitive dysfunction. In agreement with these observations, EM and Golgi staining demonstrated that synaptic loss and dendritic spine reduction were the maximal in $\delta$-secretase-injected hAPP/hMAPT mice compared with the other strains. Collectively, these findings strongly support the notion that $\delta$-secretase is sufficient for initiating AD-like pathogenesis when the substrates of APP and Tau are abundant. Remarkably, it triggers both senile plaques and NFT pathologies independent of any $\mathrm{AD}$ patient-derived mutation. This finding provides an innovative insight into the molecular mechanisms in sporadic $\mathrm{AD}$ pathogenesis. 
Since the etiology of AD is not completely known, a wide range of animal models has been developed to study the pathological processes based on APP and Tau which are two major pathological proteins found in $\mathrm{AD}$ onset ${ }^{22}$. APP is not only important for CNS maturation but also plays a role in cell contact and adhesion, in neuronal morphogenesis, in the maintenance of synaptic transmission and plasticity ${ }^{23,24}$. Accordingly, APP knockout mice develop behavioral and cognitive impairment ${ }^{25,26}$. APP overexpression results in an increased generation of toxic derivatives, $A \beta$ peptide, and/or CTFs (C-terminal fragments). Moreover, APP overproduction, either as a result of genomic locus duplication in Down Syndrome or altered regulatory sequences in the APP promoter region, leads to early-onset $\mathrm{AD}$ in humans ${ }^{27,28}$. To explore the pathological roles of human APP in AD, several transgenic mice with wild-type human APP gene have been developed. For instance, Mucke and his colleagues reported that human wild-type APP overexpression mice (hAPP $\mathrm{wt}_{\mathrm{wt}}$ I5) do not develop plaques, but show significant decreases in SYN-IR (synaptophysin-immunoreactivity) presynaptic terminals that worsen with age. Thus, FAD (familial AD) mutations are not required for the decrease in SYN-IR presynaptic terminals in hAPP mice, consistent with the loss of these structures in sporadic $A D$ patients, who also lack FAD mutations. Loss of SYN-IR presynaptic terminals and the number of NFT in specific brain regions correlate well with cognitive decline in $\mathrm{AD}^{29,30}$. Moreover, Frenchilla et al. also report that hAPP transgenic mice display early memory deficits in mice overexpressing wildtype human APP despite almost undetectable A $\beta 42$ levels in the hippocampus. However, an early increase in the levels of p-Tau in the hippocampus is manifested. In these mice, hAPP processing is non-amyloidogenic, with high levels of APP CTFs, C83, and APP intracellular domain (AICD). Furthermore, these mice present a loss of synapse-associated proteins. Importantly, signs of neurodegeneration are found in the hippocampal CA1 subfield and in the entorhinal cortex that is associated with a marked loss of MAP2 immunoreactivity, consistent with our observations described in Figs. 1 and 2. Not only is the number of neurons declined, but also the dendritic staining patterns are impaired. Immunostaining of MAP2 shows the number of branches negatively correlates with the AEP activation. This phenomenon is consistent with the results of Golgi staining which detects the dendritic spines. Conversely, in mice expressing mutant hAPP, high levels of A $\beta 42$ are found in the hippocampus, but no signs of neurodegeneration are apparent ${ }^{31}$. The results support the notion of $A \beta$-independent pathogenic pathways in $A D$. It has been proposed that $A \beta$ is synaptotoxic even in the absence of plaques and that high levels of $\mathrm{A} \beta 42$ are insufficient to induce plaque formation in mice expressing wild-type hAPP ${ }^{18}$. However, when we injected $\delta$-secretase into the hippocampus of hAPP or hAPP/hMAPT transgenic mice, we observed not only human and mouse $A \beta$ escalation (Fig. 3) but also senile plaques deposition in both mouse strains (Fig. 2), suggesting the overproduction of $A \beta$ is prone to plaque formation and deposition.

Abnormal accumulation of wild-type human Tau proteins is a hallmark of sporadic $\mathrm{AD}^{32,33}$. Expression of fulllength human Tau alone causes intracellular Tau pathologies and behavioral deficits in mice $\mathrm{e}^{19,34}$ while turning off Tau expression attenuates the pathologies ${ }^{35}$. hMAPT (htau) mice undergo an age-related accumulation of AD-relevant phosphorylation on Tau in the cell bodies and dendrites of neurons and aggregated paired helical filaments (PHF) without exogenous factors ${ }^{19}$. Accumulation of hyperphosphorylated Tau begins by 6 months but increases further by 13 and 15 months of age. Aggregated Tau and PHFs are detectable in htau mice aged 9 months via the sarkosyl-insolubility assay and immunoelectron microscopy ${ }^{19}$. It is worth noting that some of Tau N368 resided in the nuclei (Fig. 2B). Nuclear tauopathy is believed to be highly correlated to neuronal degeneration $^{36}$. Our Tau N368 antibody recognized the Tau N368 fragment generated by AEP cleavage, indicating the degeneration of CA1 neurons as well. We made similar observations in our previous report ${ }^{9}$. It should be pointed out that hMAPT mice lack endogenous mouse Tau. It has been proposed before that $4 \mathrm{R}$ mouse Tau protects htau overexpression in wild-type mice (8c mice) from developing NFT pathology that results in hMAPT from the excess of human $3 \mathrm{R}$ Tau. Nevertheless, in our hAPP/ hMAPT double-transgenic mice, they also contain mouse Tau as well, like $8 \mathrm{c}$ mice. It is worth noting that htau mice do express the first 32 amino acids of the endogenous mouse tau; however, human tau-driven tauopathy overwhelmed the contribution of the endogenous mouse tau. We observed robust NFT pathology in 5 month-old hMAPT and hAPP/hMAPT mice, when $\delta$-secretase is overexpressed. By contrast, these phenomena are alleviated in hAPP/hMAPT mice without overexpressing $\delta$-secretase (Fig. 1). Therefore, these findings strongly support that $\delta$-secretase is required for initiating AD-like pathologies, even though both wild-type human APP and human Tau are amply upregulated, highlighting that $\delta$-secretase plays a key role in the initiation of $\mathrm{AD}$ pathogenesis.

\section{Conclusion}

Currently, all AD mouse models are derived from APP, PS1, or Tau with patient-derived mutation, though familial $\mathrm{AD}$ cases are only $1 \%$ of total $\mathrm{AD}$ patients, and most $\mathrm{AD}$ patients are sporadic without any mutation. To address this 
need, we generated a human wild-type APP/MAPT double transgenic (hAPP/hMAPT) mouse model. Previously we have reported that $\delta$-secretase cleaves APP and Tau and escalating $A \beta$ and Neurofibrillary tangle (NFT) pathologies in $\mathrm{AD}$ pathologies. In this study, injection of $\delta$-secretase into hAPP/hMAPT double transgenic mice elicits $\mathrm{AD}$ pathologies in the absence of any human patient-derived mutations, supporting that $\delta$-secretase is sufficient to accelerate AD pathogenesis if both human wild-type APP and Tau substrates are sufficient. Thus, this innovative study demonstrates that $\delta$-secretase plays a key role in driving $\mathrm{AD}$ onset.

\section{Acknowledgements \\ We are thankful for Dr. Arthur W. English at Emory University for critical proofreading the manuscript and providing a lot of valuable advice. We thank Dr. L. Mucke at Gladstone Institute of Neurological Disease for providing the valuable hAPP wt 15 mice. This study was supported in part by the Rodent Behavioral Core (RBC), which is subsidized by the Emory University School of Medicine and is one of the Emory Integrated Core Facilities. Additional support was provided by the Viral Vector Core of the Emory Neuroscience NINDS Core Facilities (P30NS055077). Further support was provided by the Georgia Clinical \& Translational Science Alliance of the National Institutes of Health under Award Number UL1TR002378. This work was supported by grants from NIH grant (RF1, AG051538) to K.Y., the Funds for International Cooperation and Exchange of the NSFC (81810001048), the National Basic Research Program of China (No. 2016YFA0100800) to L.C., Shanghai Pujiang Program (No. 19PJ1409200) and the National Natural Science Foundation of China (No. 82071370) to Z.W.}

\section{Author details}

'Department of Pathology and Laboratory Medicine, Emory University School of Medicine, Atlanta, GA 30322, USA. ${ }^{2}$ Division of Spine, Department of Orthopedics, Tongji Hospital affiliated to Tongji University School of Medicine, Shanghai 200065, China. ${ }^{3}$ Key Laboratory of spine and spinal cord injury repair and regeneration, Ministry of Education of the People's Republic of China, Shanghai 200072, China

\section{Author contributions}

K.Y. conceived the project, designed the experiments, analyzed the data, and wrote the manuscript. Z.W. designed and performed most of the experiments. X.L. assessed with animal experiments. L.C. assisted with data analysis and interpretation. All authors read and approved the final manuscript.

\section{Availability of data and materials}

The datasets used and/or analyzed during the current study are available from the corresponding authors on reasonable request.

\section{Ethics approval and consent to participate}

The protocol was reviewed and approved by the Emory Institutional Animal Care and Use Committee.

\section{Conflict of interest}

The authors declare that they have no conflict of interest.

\section{Publisher's note}

Springer Nature remains neutral with regard to jurisdictional claims in published maps and institutional affiliations.

Supplementary Information accompanies this paper at (https://doi.org/ 10.1038/s41419-020-03270-7).

Received: 10 August 2020 Revised: 18 November 2020 Accepted: 19 November 2020

Published online: 12 December 2020

\section{References}

1. Selkoe, D. J. The molecular pathology of Alzheimer's disease. Neuron 6 , 487-498 (1991).

2. Heneka, M. T. et al. Neuroinflammation in Alzheimer's disease. Lancet Neurol. 14, 388-405 (2015).

3. Perl, D. P. Neuropathology of Alzheimer's disease. Mt Sinai J. Med 77, 32-42 (2010).

4. Padurariu, M., Ciobica, A., Mavroudis, I., Fotiou, D. \& Baloyannis, S. Hippocampal neuronal loss in the CA1 and CA3 areas of Alzheimer's disease patients. Psychiatr. Danub 24, 152-158 (2012).

5. Hardy, J. A. \& Higgins, G. A. Alzheimer's disease: the amyloid cascade hypothesis. Science 256, 184-185 (1992).

6. Karran, E., Mercken, M. \& De Strooper, B. The amyloid cascade hypothesis for Alzheimer's disease: an appraisal for the development of therapeutics. Nat. Rev. Drug Disco. 10, 698-712 (2011).

7. Herrup, K. The case for rejecting the amyloid cascade hypothesis. Nat. Neurosci. 18, 794-799 (2015)

8. Wu, Z. et al. Traumatic brain injury triggers APP and Tau cleavage by deltasecretase, mediating Alzheimer's disease pathology. Prog. Neurobiol. 185, 101730 (2019).

9. Zhang, Z. et al. Cleavage of tau by asparagine endopeptidase mediates the neurofibrillary pathology in Alzheimer's disease. Nat. Med 20, 1254-1262 (2014).

10. Zhang, Z. et al. Delta-secretase cleaves amyloid precursor protein and regulates the pathogenesis in Alzheimer's disease. Nat. Commun. 6, 8762 (2015).

11. Wang, Z. H. et al. C/EBPbeta regulates delta-secretase expression and mediates pathogenesis in mouse models of Alzheimer's disease. Nat. Commun. 9, 1784 (2018).

12. $\mathrm{Wu}$, Z. et al. C/EBP $\beta / \delta$-secretase signaling mediates Parkinson's disease pathogenesis via regulating transcription and proteolytic cleavage of asynuclein and MAOB. Mol. Psychiatry, https://doi.org/10.1038/s41380-020-06877 (2020).

13. Zhang, Z. et al. $\delta$-Secretase-cleaved Tau stimulates $A \beta$ production via upregulating STAT1-BACE1 signaling in Alzheimer's disease. Mol. Psychiatry, https:// doi.org/10.1038/s41380-018-0286-z (2018).

14. Wang, Z. H. et al. Delta-secretase phosphorylation by SRPK2 enhances its enzymatic activity, provoking pathogenesis in Alzheimer's disease. Mol. Cell 67, 812-825 e815 (2017).

15. Wang, Z. H. et al. BDNF inhibits neurodegenerative disease-associated asparaginyl endopeptidase activity via phosphorylation by AKT. JCI Insight 3, e99007 (2018).

16. Leuzy, A. et al. Longitudinal tau and metabolic PET imaging in relation to novel CSF tau measures in Alzheimer's disease. Eur. J. Nucl. Med. Mol. Imaging 46, 1152-1163 (2019)

17. Giannakopoulos, P. et al. Tangle and neuron numbers, but not amyloid load, predict cognitive status in Alzheimer's disease. Neurology 60, 1495-1500 (2003).

18. Mucke, L. et al. High-level neuronal expression of abeta 1-42 in wild-type human amyloid protein precursor transgenic mice: synaptotoxicity without plaque formation. J. Neurosci. 20, 4050-4058 (2000).

19. Andorfer, C. et al. Hyperphosphorylation and aggregation of tau in mice expressing normal human tau isoforms. J. Neurochem 86, 582-590 (2003).

20. Bussiere, T. et al. Morphological characterization of Thioflavin-S-positive amyloid plaques in transgenic Alzheimer mice and effect of passive Abeta immunotherapy on their clearance. Am. J. Pathol. 165, 987-995 (2004).

21. Dupont, W. D. \& Plummer, W. D. Jr. Power and sample size calculations. A review and computer program. Control Clin. Trials 11, 116-128 (1990).

22. Fabin Han, W. W. C. C. Research progress in animal models and stem cell therapy for Alzheimer's disease. J. Neurorestoratology 3, 11-22 (2015).

23. Alpar, A., Ueberham, U., Bruckner, M. K., Arendt, T. \& Gartner, U. The expression of wild-type human amyloid precursor protein affects the dendritic phenotype of neocortical pyramidal neurons in transgenic mice. Int J. Dev. Neurosci. 24, 133-140 (2006)

24. Priller, C. et al. Synapse formation and function is modulated by the amyloid precursor protein. J. Neurosci. 26, 7212-7221 (2006).

25. Dawson, G. R. et al. Age-related cognitive deficits, impaired long-term potentiation and reduction in synaptic marker density in mice lacking the beta-amyloid precursor protein. Neuroscience 90, 1-13 (1999). 
26. Phinney, A. L. et al. No hippocampal neuron or synaptic bouton loss in learning-impaired aged beta-amyloid precursor protein-null mice. Neuroscience 90, 1207-1216 (1999).

27. Cabrejo, L. et al. Phenotype associated with APP duplication in five families. Brain 129, 2966-2976 (2006).

28. Rovelet-Lecrux, A. et al. APP locus duplication causes autosomal dominant early-onset Alzheimer disease with cerebral amyloid angiopathy. Nat. Genet 38, 24-26 (2006).

29. Masliah, E., Terry, R. D., Alford, M., DeTeresa, R. \& Hansen, L. A. Cortical and subcortical patterns of synaptophysinlike immunoreactivity in Alzheimer's disease. Am. J. Pathol. 138, 235-246 (1991).

30. Honer, W. G., Dickson, D. W., Gleeson, J. \& Davies, P. Regional synaptic pathology in Alzheimer's disease. Neurobiol. Aging 13, 375-382 (1992).

31. Simon, A. M. et al. Overexpression of wild-type human APP in mice causes cognitive deficits and pathological features unrelated to Abeta levels. Neurobiol. Dis. 33, 369-378 (2009).
32. Grundke-lqbal, I. et al. Abnormal phosphorylation of the microtubuleassociated protein tau (tau) in Alzheimer cytoskeletal pathology. Proc. Natl Acad. Sci. USA 83, 4913-4917 (1986).

33. Wu, X. L., Pina-Crespo, J., Zhang, Y. W., Chen, X. C. \& Xu, H. X. Tau mediated neurodegeneration and potential implications in diagnosis and treatment of Alzheimer's disease. Chin. Med. J. 130, 2978-2990 (2017)

34. Van der Jeugd, A. et al. Hippocampal tauopathy in tau transgenic mice coincides with impaired hippocampus-dependent learning and memory, and attenuated late-phase long-term depression of synaptic transmission. Neurobiol. Learn Mem. 95, 296-304 (2011).

35. Boutajangout, A., Quartermain, D. \& Sigurdsson, E. M. Immunotherapy targeting pathological tau prevents cognitive decline in a new tangle mouse model. J. Neurosci. 30, 16559-16566 (2010).

36. Sultan, A. et al. Nuclear tau, a key player in neuronal DNA protection. J. Biol. Chem. 286, 4566-4575 (2011). 